\title{
Suppression of cellular adhesion and the anticancer activity of Aralia elata extract
}

\author{
Je-Hyuk LEE ${ }^{1 *}$
}

\begin{abstract}
The aim of this study was to provide a scientific basis for anti-arthritic and anticancer activities by inhibiting cellular adhesion molecule (CAM) expression by ingestion of Aralia elata (Miq.) Seem (A. elata), which is used in traditional medicine in East Asia. A. elata extract inhibited the adhesion between monocytic THP-1 and human umbilical vein endothelial cells (HUVEC) monolayers, respectively, compared to the TNF- $\alpha$-treated group. The methanol extract of $A$. elata potently suppressed TNF- $\alpha$ stimulated expression of vascular cell adhesion molecule-1, intercellular adhesion molecule-1, and E-selectin. Additionally, the methanol, ethyl acetate, and chloroform extracts of A. elata exhibited significant cytotoxicity against stomach cancer, melanoma, and ovarian cancer cells; however, the butanol and aqueous extracts of $A$. elata were cytotoxic only against stomach cancer cells. A. elata is anticipated to inhibit atherosclerosis, rheumatoid arthritis, and cancer progression by suppressing the expression of CAMs in HUVECs
\end{abstract}

Keywords: cellular adhesion; THP-1; HUVEC; anticancer; Aralia elata.

Practical Application: A potential suppressor on CAMs and anticancer activity of Aralia elata.

\section{Introduction}

Aralia elata (Miq) Seem (A. elata) is widely distributed in Korea, Japan, and China. In spring, the young shoots of A. elata are used in salads, soups, and dumplings in East Asia. The bark of the stem and cortex of A. elata has been used in traditional medicine for the treatment of neurasthenia, diabetes mellitus, rheumatoid arthritis, hepatitis, and gastrospasm (Li et al., 2015; Luo et al., 2015). However, until now, studies have been conducted on anti-inflammatory and anti-cancer studies of a single substance isolated from A. elata, not A. elata extract. Triterpenoid saponins isolated from A. elata reportedly inhibit acute or chronic inflammation and improve liver function (Lee et al., 2009; Luo et al., 2015). In addition, triterpene saponins and triterpene glycosides have been isolated from A. elata and have been shown to exert cytotoxicity against hepatoma, ovarian carcinoma, lung cancer (A549), promyelocytic leukemia (HL-60), and human prostate cancer (DU-145) cells (Kuang et al., 2013; Zhang et al., 2013). The aralin, isolated from A. elata, has been reported to exert anticancer activity against cervical, bladder, pancreatic, stomach, liver, and ovarian cancer cells (Tomatsu et al., 2003). However, an extract of $A$. elata has been used as a therapeutic agent for various diseases, and the studies on the anti-inflammatory and anticancer activity of $A$. elata extract are insufficient.

Blood circulation is disturbed by the formation of plaques with monocytes and lipids in the inflammation of blood vessels in coronary atherosclerosis (Libby et al., 2002). Human umbilical vein endothelial cells (HUVECs) and monocytes are involved in the progression of atherosclerosis (Blankenberg et al., 2003). Cellular adhesion between HUVECs and monocytes/neutrophils in blood vessels occurs at an early stage of atherosclerosis and rheumatoid arthritis, and is considered a target mechanism for the prevention of atherosclerosis (Galkina \& Ley, 2007). Cellular adhesion molecules (CAMs), such as vascular cell adhesion molecule-1 (VCAM-1), intracellular adhesion molecule-1 (ICAM-1), and E-selectin, are expressed in HUVECs and stimulate adhesion with monocytes or neutrophils in the blood (Iiyama et al., 1999; Blankenberg et al., 2003).

The aim of this study was to investigate the inhibitory activity of the A. elata extract on the adhesion between HUVECs and monocytes, and its anticancer activity. The results of this study suggest that $A$. elata extract may inhibit atherosclerosis and rheumatoid arthritis and exhibit anticancer activity.

\section{Materials and methods}

\subsection{Chemicals and reagents}

Calcein $O, O^{\prime}$-diacetate tetrakis (acetoxymethyl) ester (CalceinAM), 3-(4,5-dimethylthiazol-2-yl)-2,5-diphenyltetrazolium bromide (MTT), and dimethyl sulfoxide (DMSO) were purchased from Sigma-Aldrich (St. Louis, MO, USA). F-12K, RPMI-1640, fetal bovine serum (FBS), penicillin/streptomycin, endothelial cell growth supplement (ECGS), and trypsin-EDTA required for cell culture were purchased from GIBCO (Invitrogen Inc., Grand Island, NY, USA). Tumor necrosis factor- $\alpha$ (TNF- $\alpha$ ) was purchased from BD Science (San Jose, CA, USA), and other materials were of analytical grade.

\subsection{Preparation of A. elata extracts}

The root bark of $A$. elata was purchased from traditional medicine markets in Seoul, Korea. A. elata root bark extraction 
was performed using $70 \%$ methanol for $24 \mathrm{~h}$ at $30{ }^{\circ} \mathrm{C}$, and the impurities in the crude extract were removed by filtration through Whatman paper No. 1. It was then concentrated under reduced pressure using a rotary evaporator (EYELA, NY, USA). The methanol extract of $A$. elata was redissolved in distilled water at $30{ }^{\circ} \mathrm{C}$ and was further subjected to continuous extraction using chloroform, ethyl acetate, $n$-butanol, and water. All extract fractions $(100 \mathrm{mg} / \mathrm{mL})$ were dissolved in DMSO at $-20^{\circ} \mathrm{C}$ and used for cell culture.

\subsection{Cell culture}

Skin cancer cells (melanoma B16-F1, CRL-6323) were purchased from the American Type Culture Collection (ATCC, Manassas, VA, USA) and cultured in the Dulbecco's modified Eagle medium (DMEM, Life Technologies, GIBCO). Stomach cancer cells (SNU-638 and AGS), ovarian cancer cells (adenocarcinoma, NIH: OVCAR-3), and monocytes (THP-1) were purchased from the Korean Cell Line Bank (KCLB) and cultured in the RPMI-1640 medium. All media were supplemented with 10\% (v/v) FBS and $100 \mathrm{U} / \mathrm{mL}$ penicillin/streptomycin.

Floating THP-1 cells were collected by centrifugation for $2 \mathrm{~min}$ at 2,090 $\times g$ and were suspended in fresh RPMI-1640 medium for subculture. Human vein umbilical endothelial cells (HUVECs, CRL-2480, ATCC) were cultured using F-12K nutrient mixtures with $0.1 \mathrm{mg} / \mathrm{mL}$ of heparin and $0.03 \mathrm{mg} / \mathrm{mL}$ of ECGS. All cell lines were cultured at $37^{\circ} \mathrm{C}$ in a $5 \% \mathrm{CO}_{2}$ humidified incubator (MCO-18AC, Panasonic Healthcare Co., Ltd., Japan). For the subculture, all cell lines except THP-1 were subjected to washing steps twice with $\mathrm{PBS}$ ( $\mathrm{pH}$ 7.4) to remove the serum containing a trypsin inhibitor and were separated using 0.05\% trypsin containing $0.53 \mathrm{mM}$ EDTA from the bottom of the culture flask; thereafter, they were fractionated with fresh medium, and subjected to passages. HUVECs and THP-1 cells were used at passage numbers 20-30 for cellular adhesion study.

\subsection{Cell viability assay}

Cell viability in the presence of the A. elata extracts was assessed using a modified MTT colorimetric assay. THP-1 $\left(1 \times 10^{4}\right.$ cells/well), HUVECs $\left(5 \times 10^{3}\right.$ cells/well $)$, and cancer cell lines $\left(1 \times 10^{4}\right.$ cells/well) were seeded into 96 -well tissue culture plates (Corning Inc., USA) and cultivated at $37{ }^{\circ} \mathrm{C}$ for $24 \mathrm{~h}$. Then, A. elata extracts were added to the cultured cells. After 24 and $48 \mathrm{~h}$ of cultivation for cancer cells and THP-1/HUVECs, respectively, MTT $(0.5 \mathrm{mg} / \mathrm{mL})$ was added to all wells, and cells were further incubated for $4 \mathrm{~h}$. After all media were carefully discarded from the culture plate, $100 \mu \mathrm{L}$ of DMSO was added to the wells and mixed by shaking for $5 \mathrm{~min}$ to dissolve the purple formazan crystals formed in the cells. The level of MTT formazan in DMSO was measured at $540 \mathrm{~nm}$ using a microplate reader (SPARK 10M, Tecan Inc., Grŏdig, Austria). Cell viability was expressed as the ratio of absorbance of MTT formazan between the A.elata extract-treated cells and untreated cells.

\subsection{Quantification of THP-1 cell attachment to endothelial cells}

HUVECs were seeded in black 24-well culture plates (Corning 4445 , Corning Inc., NY, USA) at a density of $5 \times 10^{4}$ cells/well and incubated at $37^{\circ} \mathrm{C}$ for $24 \mathrm{~h}$. After adding the A. elata extracts to a fully cultivated HUVEC monolayer, TNF- $\alpha(5 \mathrm{ng} / \mathrm{mL})$ was added to stimulate the expression of CAM genes in HUVEC. After further incubation for $24 \mathrm{~h}$, HUVEC monolayers were subjected to washing steps three times with PBS. THP-1 cells labeled with calcein-AM were added to the HUVEC monolayer with $1 \times 10^{6}$ cells/well and cultivated for $1 \mathrm{~h}$. The unattached THP1 cells were removed by washing four times using PBS, and the adhesion of calcein-AM-labeled monocytes to HUVECs was determined by absorbance using a fluorescent plate reader (SPARK 10M, Tecan Inc., Grŏdig, Austria). The excitation and emission wavelengths of the calcein-AM molecules were 485 and $530 \mathrm{~nm}$, respectively. Calcein-AM-labeled THP-1 cells attached to the HUVEC monolayer were observed by imaging at a magnification of $200 \times$ with an inverted fluorescence microscope (IX 71; Olympus Inc., Tokyo, Japan) equipped with an Olympus DP50 camera. The collected photographic information was processed using an imaging software (View finder Lite, Ver. 1.0.134, Pixera Corporation, Los Gatos, CA, USA, and the OLYSIA BioAutoCell Ver. 3.2. Soft Imaging Systems, Tokyo, Japan).

\subsection{Reverse transcriptase-polymerase chain reaction ( $R T$ - PCR) for cellular adhesion molecule (CAM) genes}

Total RNA was isolated from HUVECs using an RNeasy kit (Qiagen Inc., Valencia, CA, USA). For the transcription of CAM genes using the total RNA as a template, RT-PCR was performed using the one-step RT-PCR kit (Qiagen GmbH, D-10424 Hilden, Germany), which is used to conduct the synthesis of cDNA and PCR together. The following primers $(1 \mu \mathrm{M})$ were used for PCR: Human vascular cellular adhesion molecule-1 (hVCAM-1), sense 5'ATGCCTGGGAAGATGGTCGTGA-3' and antisense 5'-TGGAGCTGGTAGACCCTCGCTG-3'); human intracellular adhesion molecule-1 (hICAM-1), sense 5'-GGTGACGCTGGTGACGCTGAATGGGGTTCC-3' and antisense 5'-GTCCTCATGGTGGGGCTATGACTC-3'; and human E-selectin, sense 5'-ATCATCCTGCAACTTCACC-3' and antisense 5'-ACACCTCACCAAACCCTTC-3' (Lee et al., 2007). The glyceraldehyde-3-phosphate dehydrogenase (GAPDH) gene, a housekeeping gene, was used as an internal reference gene to compare the transcription levels of CAM genes in HUVECs; Human GAPDH primer: sense 5'ATGACAACAGCCTCAAGATCATCAG3' and antisense 5'-CTGGTGGTCCAGGGGTCTTACTCCT-3'. The Bio-Rad thermal cycler (MJ Mini; Bio-Rad Inc., Hercules, CA, USA) for conducting reverse transcription and PCR was programmed, with slight modifications, as per methods reported by Park et al. (2003) and Choi et al. (2004). For cDNA synthesis and initial PCR activation using total RNA $(1 \mu \mathrm{g} / \mu \mathrm{L})$, the thermal cycler conditions were set to one cycle of $30 \mathrm{~min}$ at $50^{\circ} \mathrm{C}$ and $15 \mathrm{~min}$ at $95^{\circ} \mathrm{C}$. For amplification of target genes, the thermal cycler was programmed for 30 cycles of denaturation at $95^{\circ} \mathrm{C}$ for $1 \mathrm{~min}$, annealing at $55^{\circ} \mathrm{C}$ for $2 \mathrm{~min}$, and extension at $72^{\circ} \mathrm{C}$ for $3 \mathrm{~min}$, and for one cycle of a final extension at $72^{\circ} \mathrm{C}$ for $10 \mathrm{~min}$.

\subsection{Statistical analysis}

All experiments were repeated three times and the results are presented as mean \pm standard deviation (SD). The results were analyzed using one-way analysis of variance (ANOVA) 
and Dunnett's multiple comparisons to determine the statistical significance between the experimental results. Statistically significance was considered when the $p$-value was less than 0.05 .

\section{Results and discussion}

\subsection{Cytotoxicity of A. elata against HUVECs and monocytic THP-1 cells}

The cytotoxicity of the A. elata methanol extract $(0-2,000 \mu \mathrm{g} / \mathrm{mL})$ was monitored in HUVECs and monocytic THP-1 cells (Figure 1). No cytotoxicity against THP-1 was observed in concentrations of $\leq 1,000 \mu \mathrm{g} / \mathrm{mL}$ of the A. elata methanol extract; however, approximately $45.9 \%$ of the cells exhibited damage at $2,000 \mu \mathrm{g} / \mathrm{mL}$ of A. elata methanol extract. The A. elata extract did not cause any cytotoxicity in HUVECs at concentrations ranging from $0-500 \mu \mathrm{g} / \mathrm{mL}$; however, the viability of HUVECs was decreased to approximately $16.7 \%$ and $41.2 \%$ at concentrations of 1,000 and $2,000 \mu \mathrm{g} / \mathrm{mL}$ of the $A$. elata methanol extract, respectively. Even in previous studies, the A. elata extract has not been reported to exhibit cytotoxicity against macrophages (RAW 264.7) and keratinocytes (HaCaT) at concentrations lower than 100 and $300 \mu \mathrm{g} / \mathrm{mL}$, respectively (Lee \& Jeong, 2009; Kwak \& Yang, 2016). Therefore, the concentration range $(0-500 \mu \mathrm{g} / \mathrm{mL})$ of $A$. elata extract not exhibiting cytotoxicity against normal THP-1 cells and HUVECs was considered acceptable.

\subsection{Inhibition of cellular adhesion between HUVECs and monocytic THP-1 cells by A. elata}

We investigated the inhibitory effect of the A. elata methanol extract on the intercellular adhesion between HUVECs and monocytic THP- 1 cells stimulated by TNF- $\alpha$. The adhesion of calcein-AM-labeled monocytic THP-1 cells to basal HUVECs was insignificant. The intercellular adhesion of THP-1 cells to HUVEC monolayer cells was stimulated by the addition of TNF- $\alpha$

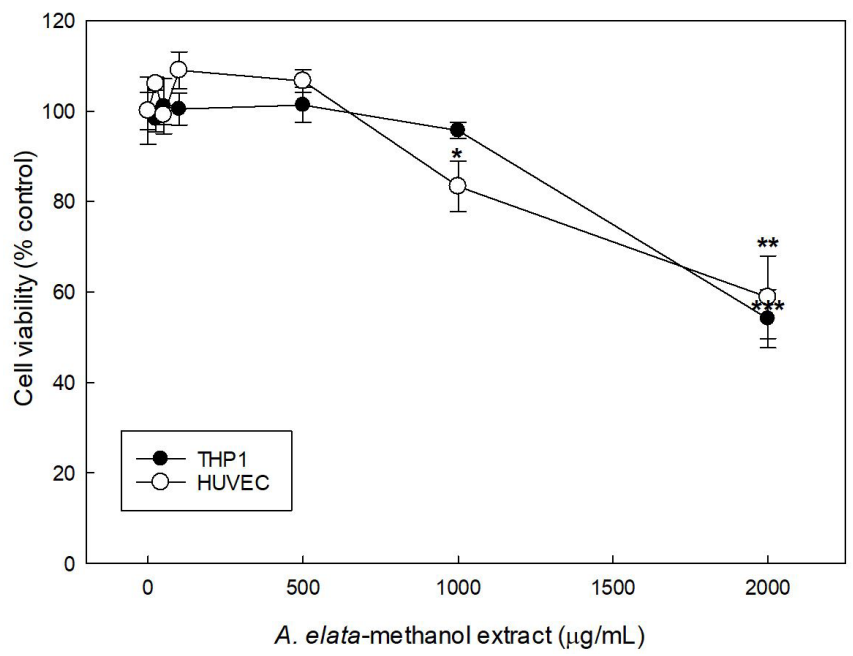

Figure 1. Viability of THP-1 cells and HUVECs in the presence of the Aralia elata methanolic extract. Cell viability was expressed in comparison with the control. ${ }^{\star} p<0.05,{ }^{* *} p<0.005$, and ${ }^{* * *} p<0.001$ depict significant differences compared to the control.
(Figure 2). A. elata methanol extract suppressed intercellular adhesion in a dose-dependent manner, whereby concentrations ranging between 100 and $500 \mu \mathrm{g} / \mathrm{mL}$ inhibited approximately $54.1 \%$ and $68.3 \%$ of the adhesion between THP- 1 and HUVEC monolayers, respectively, compared to the TNF- $\alpha$-treated group.

The adherence of monocytes to damaged sites in HUVEC monolayers is stimulated by cytokines, such as TNF- $\alpha$, and is essential for the development and progression of atherosclerosis and rheumatoid arthritis (Ludwig et al., 2004). CAMmediated adhesion of monocytes increases the rolling and transmigration of monocytes into the subendothelial space, wherein they differentiate into macrophages (Wilcox et al., 1989; Rajavashisth et al., 1990). The adherence of monocytes to HUVECs is mediated by the interaction between monocytes and CAMs in HUVECs (Ludwig et al., 2004). Eventually, macrophages, $\mathrm{T}$ lymphocytes, and vascular smooth muscle cells form plaques in blood vessels, subsequently narrowing them and resulting in the development of atherosclerosis (Iiyama et al., 1999). Therefore, attempts to suppress the cellular adhesion between monocytes and HUVECs have been reported for the prevention of inflammation, arthritis, and atherosclerosis (Grober et al., 1993; Tak et al., 1995). Tea flavonoids, black garlic extract, and Allium victorialis var. platyphyllum extract inhibit the adhesion of monocytes to HUVECs and suppress the expression of CAMs in HUVECs (Ludwig et al., 2004; Lee et al., 2007). However, the inhibition of THP-1 cell adhesion to the HUVEC monolayer by the $A$. elata extract has not been reported thus far. This study was conducted to identify the therapeutic potential of the A. elata extract in inhibiting early-stage inflammation, arthritis, and atherosclerosis.

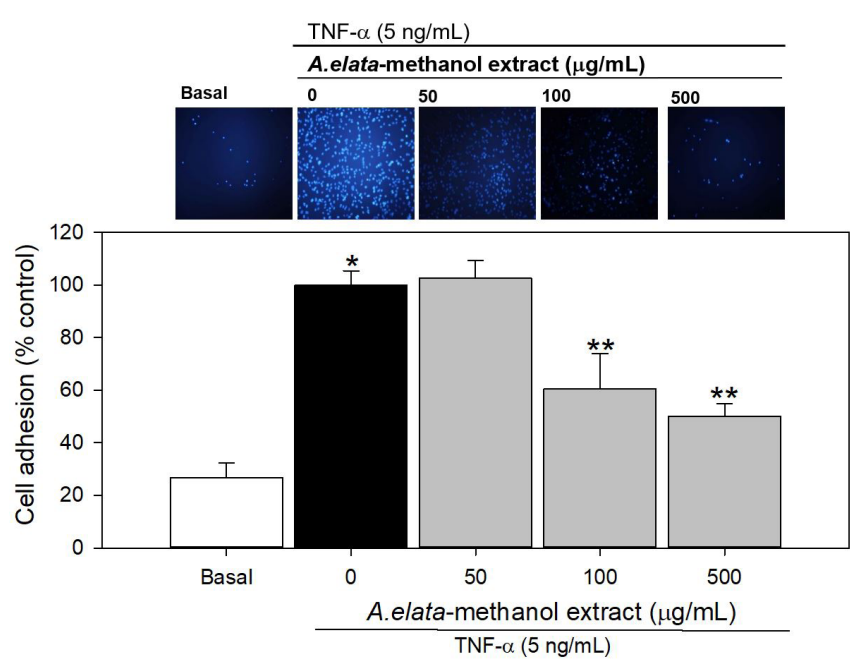

Figure 2. Inhibitory activity of the Aralia elata methanolic extract on the adhesion between monocytic THP-1 and human umbilical vein endothelial cell (HUVEC) monolayers. Calcein-AM-labeled THP-1 cells attached to HUVEC monolayers were photographed at a magnification of 200x using an inverted fluorescence microscope (IX 71; Olympus INC., Tokyo, Japan) connected to an Olympus DP50 camera with an imaging software (Viewfinder Lite, Ver. 1.0.134, Pixera Corporation, Los Gatos, CA, USA and Olysia Bioautocell ver. 3.2, Soft Imaging System, Tokyo, Japan). ${ }^{\star} p<0.05$, as compared to the basal activity, ${ }^{*} p<0.05$, as compared to the TNF- $\alpha$-treated group. 


\subsection{Inhibitory effect of A. elata on the transcription of CAMs in HUVECs}

The transcription of CAMs, stimulated by TNF- $\alpha$, causes the adhesion of THP- 1 cells to HUVECs and plays an important role in early-stage inflammation, atherosclerosis, and rheumatoid arthritis (Ludwig et al., 2004). The TNF- $\alpha$-induced transcription of VCAM-1, ICAM-1, and E-selectin was approximately 5.36, 4.87, and 7.4 times higher than that of the basal level, respectively. However, the A. elata methanol extract significantly inhibited the transcription of VCAM-1, ICAM-1, and E-selectin in HUVEC monolayers in a dose-dependent manner (Figure 3). The transcription of VCAM- 1 was decreased to 86.7 and $93.3 \%$ by the A. elata extract (50 and $100 \mu \mathrm{g} / \mathrm{mL}$ ), respectively. Particularly, at $500 \mu \mathrm{g} / \mathrm{mL}$ of the A. elata extract, VCAM-1 transcription was lower than the basal level (Figure 3A). The transcription of ICAM-1 in HUVECs was suppressed to 10.3, 10.3, and $43.1 \%$ by the A. elata extract $(50,100$, and $500 \mu \mathrm{g} / \mathrm{mL})$, respectively (Figure 3B). Additionally, 50, 100, and $500 \mu \mathrm{g} / \mathrm{mL}$ of the A. elata extract decreased the transcription levels of E-selectin to 14.1, 15.6 , and $62.5 \%$, respectively (Figure $3 \mathrm{C}$ ).

The expression of CAMs on the surface of endothelial cells activated by inflammatory stimulants is increased, resulting in the mobilization of monocytes and their differentiation into macrophages (Blankenberg et al., 2003). Atherosclerosis is a chronic inflammatory disease characterized by the formation of plaques composed of foam cells, immune cells, vascular endothelial cells, smooth muscle cells, platelets, and extracellular matrix components (Galkina \& Ley, 2007). VCAM-1 expression is induced not only in endothelial cells, but also in other cells, such as macrophages, myoblasts, and dendritic cells. Morphological changes in endothelial cells and leukocyte migration are induced by VCAM-1 (Blankenberg et al., 2003). ICAM-1 is a large immunoglobulin superfamily with membrane glycoprotein receptors that contain multiple extracellular immunoglobulin domains. ICAM-1 is expressed constitutively at low levels in leukocytes and endothelial cells, but the expression is upregulated by pro-inflammatory cytokines. ICAM-1 forms strong bonds with integrins and mediates the adhesion of monocytes to activated endothelial cells (Blankenberg et al., 2003). E-selectin is expressed specifically in endothelial cells, and is rarely expressed in resting cells. E-selectin expression is transcriptionally induced by several inflammatory cytokines, promoting the rolling and tethering of attached monocytes in endothelial cells (Blankenberg et al., 2003).

The transcription and expression of CAMs is suppressed by flavonoids (apigenin, luteolin, and epigallocatechin-3-gallate) (Gerritsen et al., 1995; Ludwig et al., 2004), grape seed extract, Allium victorialis var. platyphyllum, and Cirsium sp. extracts (Lee et al., 2007; Lee et al., 2008; Sen \& Bagchi, 2001). However, there have been few reports on the suppression of CAM transcription by $A$. elata extract, which is used as a traditional medicine in several countries, including Korea, China, and Japan. This study supports that $A$. elata extract may be used for the prevention of atherosclerosis and rheumatoid arthritis by regulating CAM expression.

\subsection{Anticancer activity of A. elata against several cancer cell lines}

The cytotoxicity of the A. elata extracts was studied in four cancer cell lines (Figure 4). Methanol, ethyl acetate, and chloroform extracts of $A$. elata exhibited the potent cytotoxicity against stomach cancers (Figure $4 \mathrm{~A}$ and $4 \mathrm{~B}$ ), melanoma (Figure $4 \mathrm{C}$ ), and ovarian cancer cells (Figure 4D) at a concentration of $200 \mu \mathrm{g} / \mathrm{mL}$; however, the butanol and aqueous extracts of $A$. elata were only cytotoxic against the SNU stomach cancer cell line. Methanol, ethyl acetate, and chloroform extracts of A. elata decreased the viability of cancer cells in a dose-dependent manner. These extracts $(200 \mu \mathrm{g} / \mathrm{mL})$ reduced the viability of SNU stomach cancer cells by approximately $12.0 \%, 25.1 \%$, and $16.0 \%$, respectively (Figure $4 \mathrm{~A}$ ). The $\mathrm{IC}_{50}$ values of $A$. elata methanol, ethyl acetate, and chloroform extracts against SNU stomach cancer cells were $62.83,153.3$, and $41.2 \mu \mathrm{g} / \mathrm{mL}$, respectively, and the chloroform extract showed the most potent inhibition of SNU cell growth. Additionally, the butanol and aqueous extracts of A. elata weakly
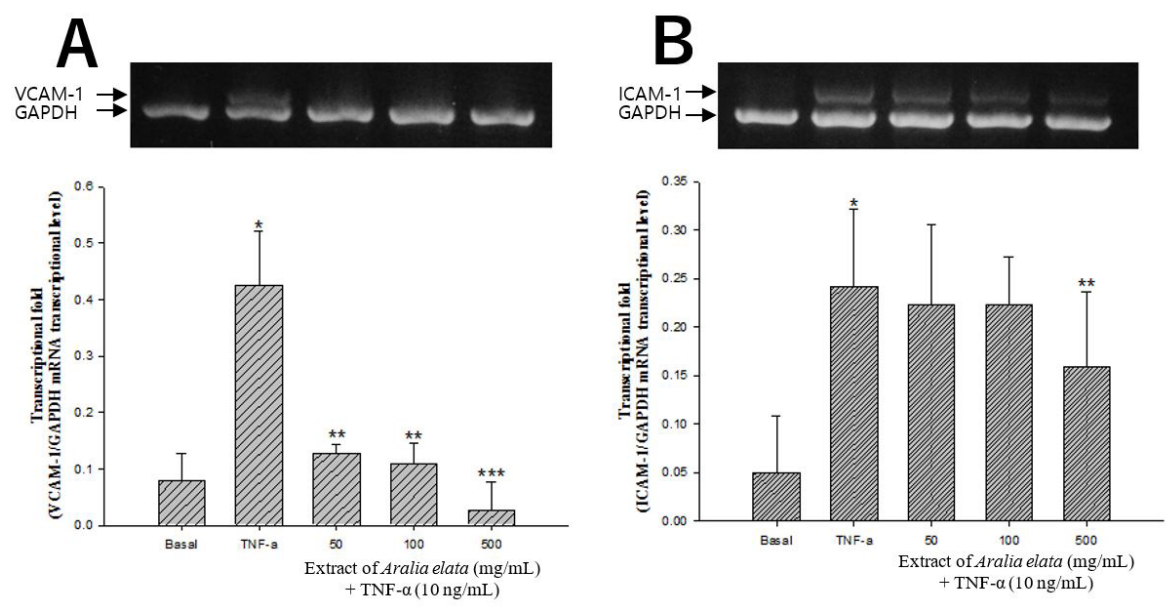
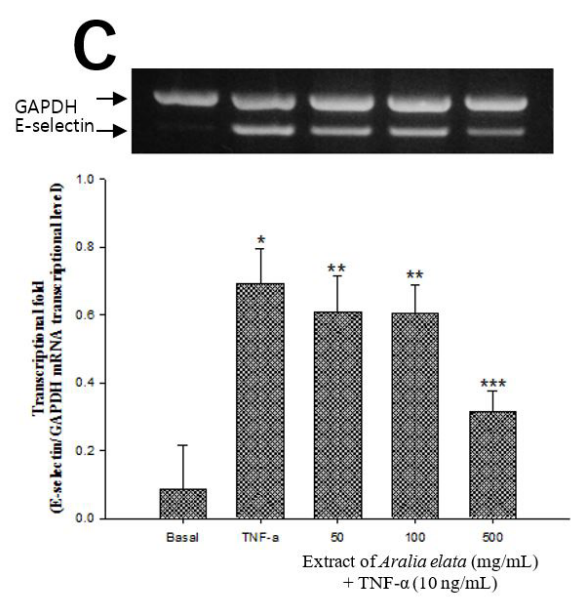

Figure 3. Suppressive activity of the Aralia elata methanolic extract on transcription of cellular adhesion molecules in HUVEC monolayer. A: VCAM-1, ${ }^{*} p<0.05$, as compared to the basal activity; ${ }^{* *} p<0.05$ and ${ }^{* * *} p<0.005$, as compared to the TNF- $\alpha$-treated group; B: ICAM-1, ${ }^{\star} p<0.05$, as compared to the basal activity; ${ }^{\star *} p<0.5$, as compared to the TNF- $\alpha$-treated group; C: E-selectin, ${ }^{\star} p<0.05$, as compared to the basal activity; ${ }^{* *} p<0.05$ and ${ }^{* * *} p<0.005$, as compared to the TNF- $\alpha$-treated group. 


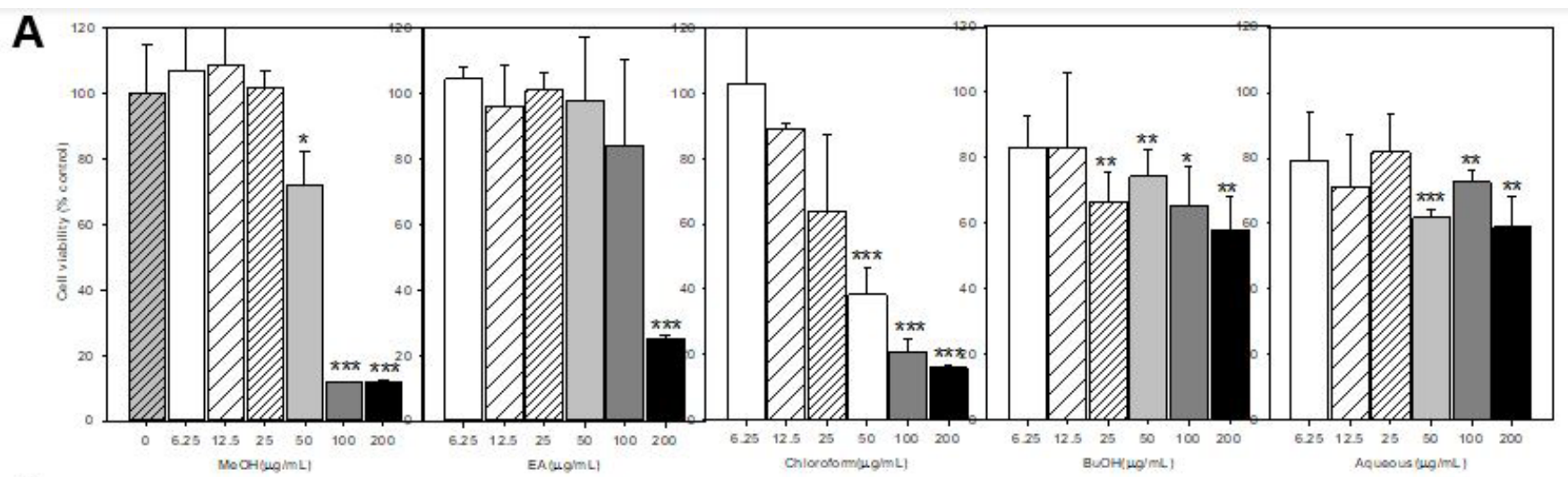

B

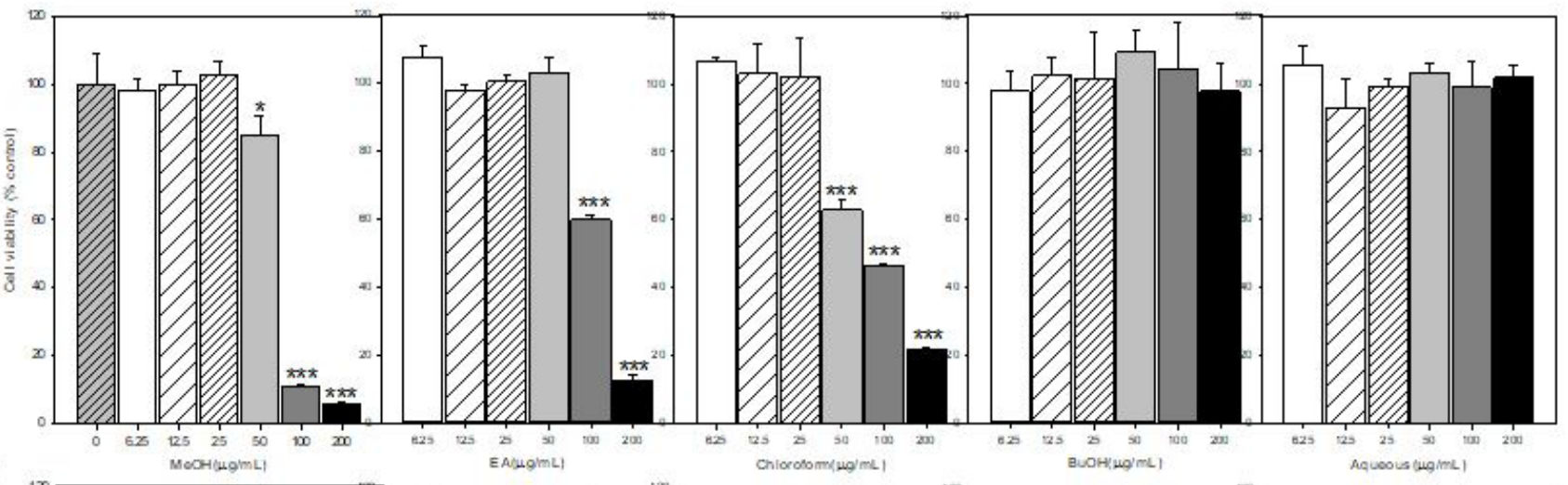

C

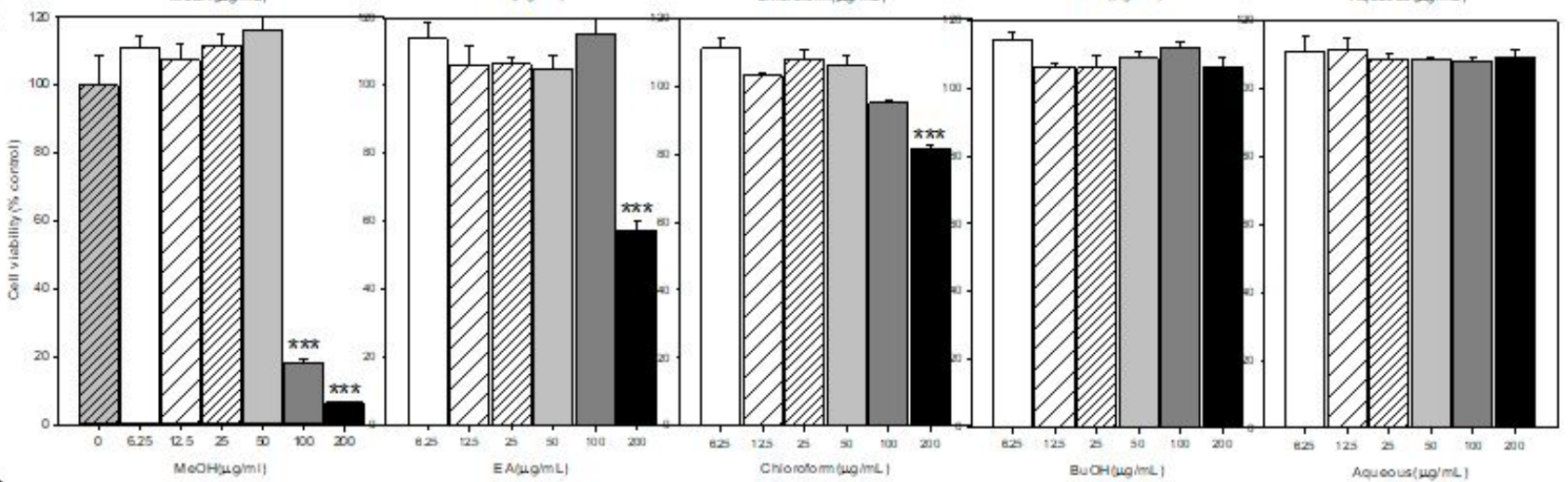

D

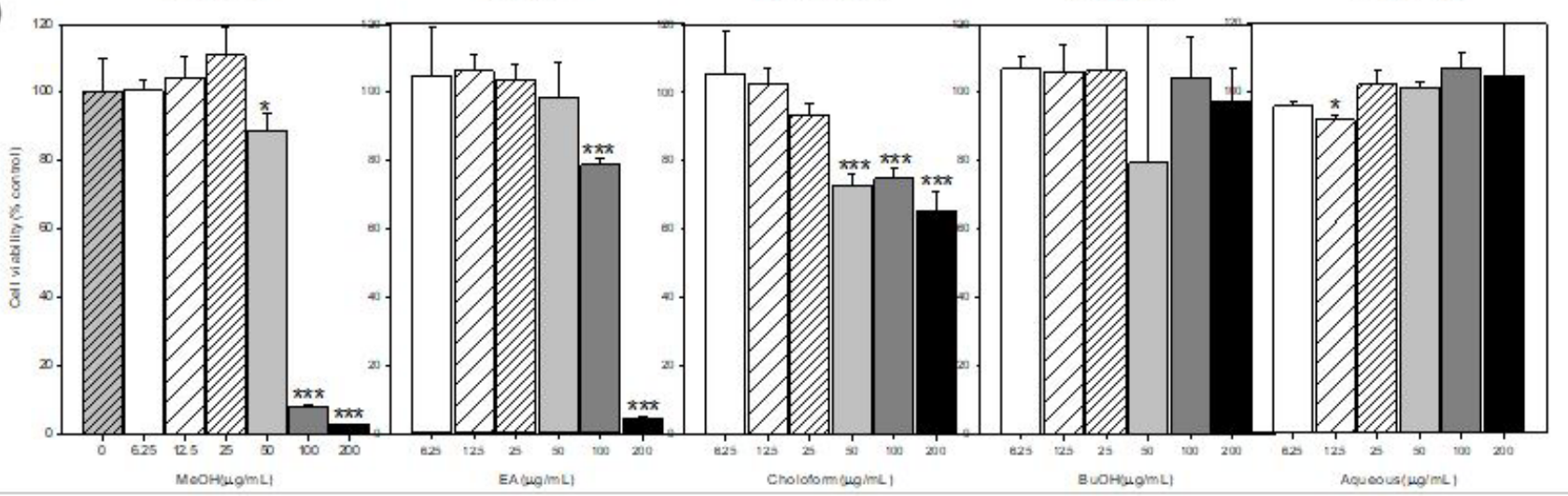

Figure 4. Anti-carcinogenic activity of the Aralia elata extract fractions. MEOH: methanol extract of A.elata; EA: ethyl acetate extract of A.elata; BUOH: butanol extract of A.elata; Aqueous: water extract of A.elata; Chroloform: chloroform extract of A.elata. Cell viability was calculated by comparison with the control group. (A) SNU: stomach cancer cell line (control $=99.99 \% \pm 15.24 \%$ ); (B) AGS: stomach cancer cell line (control = 100\% $\pm 9.15 \%)$; (C) melanocyte: skin cancer cell (control = 100.04\% $\pm 8.46 \%)$; (D) SK-OV-3: ovarian cancer cell line $($ control $=100.28 \%$ $\pm 9.90 \%) .{ }^{*} p<0.05,{ }^{* *} p<0.005$, and ${ }^{* * *} p<0.001$, compared to the control. 
inhibited the viability of SNU cancer cells (approximately 58.0 and $58.8 \%$ at $200 \mu \mathrm{g} / \mathrm{mL}$, respectively) in a dose-dependent manner. The cell viability of AGS cells was suppressed to 6.0, 12.6, and $21.7 \%$ by the methanol, ethyl acetate, and chloroform extracts $(200 \mu \mathrm{g} / \mathrm{mL})$ of A. elata, respectively, and the methanol extract was the most effective inhibitor of cell viability $\left(\mathrm{IC}_{50}=66.1 \mu \mathrm{g} / \mathrm{mL}\right)$ (Figure 4B). The methanol extract $(200 \mu \mathrm{g} / \mathrm{mL})$ of $A$. elata significantly inhibited the viability of melanoma cells by $95.6 \%$ (Figure 4C), and ethyl acetate and chloroform extracts of A. elata weakly inhibited melanoma growth. Additionally, the methanol and ethyl acetate extracts $(200 \mu \mathrm{g} / \mathrm{mL})$ of $A$. elata significantly inhibited the viability of ovarian cancer cells to $2.7 \%$ and $4.3 \%$, respectively (Figure 4D).

Numerous compounds derived from A. elata have been reported to exert anticancer activities. Triterpene saponins, aralin, and araloside A, isolated from A. elata, inhibit the growth of the kidney, cervical, bladder, and lung cancer cell lines (Tomatsu et al., 2003; Yu et al., 2011; Zhang et al., 2013). However, the anticancer properties of the whole extract of A. elata have not been studied extensively, with only a few reports on human pulmonary carcinoma, breast adenocarcinoma, and colon carcinoma (Chon et al., 2007; Shikov et al., 2016). Endothelial CAMs associated with the adhesion of cancer cells to cardiovascular epithelial cells are involved in various stages of tumor progression and metastasis (McCarthy et al., 1991; Banks et al., 1993). ICAM-1 is involved not only in the establishment of inflammation-mediated intercellular interaction, but is also involved in melanoma progression (Natali et al., 1990) and the metastasis of gastric, colon, gallbladder, and pancreatic cancers (Tsujisaki et al., 1991). E-selectin is transiently expressed in activated endothelial cells and plays a pivotal role in stimulating/ activating adhesion receptors to target circulating tumor cells to endothelial cells. This is achieved by mediating the adhesion of neutrophils, monocytes, and memory T cells (Honn \& Tang, 1992). Additionally, VCAM-1 and E-selectin are associated with the adhesion of melanoma cells and colon carcinoma cells to the endothelium, respectively (Rice \& Bevilacqua, 1989; Lauri et al., 1991).

In this study, the A. elata extracts suppressed the transcription of CAMs in HUVECs and exerted anticancer activities against four cancer cell lines. Taken together, A. elata extracts may be used to prevent atherosclerosis, rheumatoid arthritis, and cancer progression by suppressing the transcription of CAMs in HUVECs.

\section{References}

Banks, R. E., Gearing, A. J., Hemingway, I. K., Norfolk, D. R., Perren, T. J., \& Selby, P. J. (1993). Circulating intercellular adhesion molecule-1 (ICAM-1), E-selectin and vascular cell adhesion molecule-1 (VCAM1) in human malignancies. British Journal of Cancer, 68(1), 122-124. http://dx.doi.org/10.1038/bjc.1993.298. PMid:7686390.

Blankenberg, S., Barbaux, S., \& Tiret, L. (2003). Adhesion molecules and atherosclerosis. Atherosclerosis, 170(2), 191-203. http://dx.doi. org/10.1016/S0021-9150(03)00097-2. PMid:14612198.

Choi, J. S., Choi, Y. J., Park, S. H., Kang, J. S., \& Kang, Y. H. (2004). Flavones migrate tumor necrosis factor- $\alpha$-induced adhesion molecule upregulation in cultured human endothelial cells: Role of nuclear factor- $\kappa$ B. The Journal of Nutrition, 134(5), 1013-1019. http://dx.doi. org/10.1093/jn/134.5.1013. PMid:15113938.

Chon, S. U., Ahn, C. Y., \& Lee, S. Y. (2007). In vitro assay on antioxidant activity and cytotoxicity of methanol extracts from young sprouts of several Korean salad plants. Korean Journal of Plant Resources, 20(6), 499-503.

Galkina, E., \& Ley, K. (2007). Vascular adhesion molecules in atherosclerosis. Arteriosclerosis, Thrombosis, and Vascular Biology, 27(11), 2292-2301. http://dx.doi.org/10.1161/ATVBAHA.107.149179. PMid:17673705.

Gerritsen, M. E., Carley, W. W., Ranges, G. E., Shen, C. P., Phan, S. A., Ligon, G. F., \& Perry, C. A. (1995). Flavonoids inhibit cytokineinduced endothelial cell adhesion protein gene expression. American Journal of Pathology, 147(2), 278-292. PMid:7543732.

Grober, J. S., Bowen, B. L., Ebling, H., Athey, B., Thompson, C. B., Fox, D. A., \& Stoolman, L. M. (1993). Monocyte-endothelial adhesion in chronic rheumatoid arthritis. In situ detection of selectin and integrindependent interactions. The Journal of Clinical Investigation, 91(6), 2609-2619. http://dx.doi.org/10.1172/JCI116500. PMid:7685772.

Honn, K. V., \& Tang, D. G. (1992). Adhesion molecules and tumor cell interaction with endothelium and subendothelial matrix. Cancer and Metastasis Reviews, 11(3-4), 353-375. http://dx.doi.org/10.1007/ BF01307187. PMid:1423822.

Iiyama, K., Hajra, L., Iiyama, M., Li, H., Dichiara, M., Medoff, B. D., \& Cybulsky, M. I. (1999). Patterns of vascular cell adhesion molecule-1 and intercellular adhesion molecule- 1 expression in rabbit and mouse atherosclerotic lesions and at sites predisposed to lesion formation. Circulation Research, 85(2), 199-207. http:// dx.doi.org/10.1161/01.RES.85.2.199. PMid:10417402.

Kuang, H. X., Wang, Z. B., Wang, Q. H., Yang, B. Y., Xiao, H. B., Okada, Y., \& Okuyama, T. (2013). Triterpene glucosides from the leaves of Aralia elata and their cytotoxic activities. Chemistry \& Biodiversity, 10(4), 703-710. http://dx.doi.org/10.1002/cbdv.201200087. PMid:23576356.

Kwak, C. S., \& Yang, J. (2016). Suppressive effects of ethanol extract of Aralia elata on UVB-induced oxidative stress in human keratinocytes. Journal of Nutrition and Health, 49(3), 135-143. http://dx.doi. org/10.4163/jnh.2016.49.3.135.

Lauri, D., Needham, L., Martin-Padura, I., \& Dejana, E. (1991). Tumor cell adhesion to endothelial cells: Endothelial leukocyte adhesionmolecule- 1 as an inducible adhesive receptor specific for colon carcinoma cells. Journal of the National Cancer Institute, 83(18), 1321-1324. http://dx.doi.org/10.1093/jnci/83.18.1321. PMid:1715924.

Lee, J. H., Choi, S. I., Lee, Y. S., \& Kim, G. H. (2007). Antioxidant and anti-inflammatory activities of Allium victorialis subsp. platyphyllum extracts. Food Science and Biotechnology, 16(5), 796-801.

Lee, J. H., Choi, S. I., Lee, Y. S., \& Kim, G. H. (2008). Antioxidant and anti-inflammatory activities of ethanol extract from leaves of Cirsium japonicum. Food Science and Biotechnology, 17(1), 38-45.

Lee, J. H., Ha, Y. W., Jeong, C. S., Kim, Y. S., \& Park, Y. (2009). Isolation and tandem mass fragmentations of an anti-inflammatory compound from Aralia elata. Archives of Pharmacal Research, 32(6), 831-840. http://dx.doi.org/10.1007/s12272-009-1603-5. PMid:19557359.

Lee, J. H., \& Jeong, C. S. (2009). Suppressive effects on the biosynthesis of inflammatory mediators by Aralia elata extract fractions in macrophage cells. Environmental Toxicology and Pharmacology, 28(3), 333-341. http://dx.doi.org/10.1016/j.etap.2009.05.009. PMid:21784024.

Li, F., He, X., Niu, W., Feng, Y., Bian, J., \& Xiao, H. (2015). Acute and sub-chronic toxicity study of the ethanol extract from leaves of Aralia elata in rats. Journal of Ethnopharmacology, 175, 499-508. http:// dx.doi.org/10.1016/j.jep.2015.10.002. PMid:26456344. 
Libby, P., Ridker, P. M., \& Maseri, A. (2002). Inflammation and atherosclerosis. Circulation, 105(9), 1135-1143. http://dx.doi. org/10.1161/hc0902.104353. PMid:11877368.

Ludwig, A., Lorenz, M., Grimbo, N., Steinle, F., Meiners, S., Bartsch, C., Stangl, K., Baumann, G., \& Stangl, V. (2004). The tea flavonoid epigallocatechin-3-gallate reduces cytokine-induced VCAM-1 expression and monocyte adhesion to endothelial cells. Biochemical and Biophysical Research Communications, 316(3), 659-665. http:// dx.doi.org/10.1016/j.bbrc.2004.02.099. PMid:15033450.

Luo, Y., Dong, X., Yu, Y., Sun, G., \& Sun, X. (2015). Total aralosides of Aralia elata (Miq.) seem (TASAES) ameliorate nonalcoholic steatohepatitis by modulating IRE1 $\alpha$-mediated JNK and NF- $\kappa \mathrm{B}$ pathways in ApoE-/-mice. Journal of Ethnopharmacology, 163, 241-250. http://dx.doi.org/10.1016/j.jep.2015.01.017. PMid:25655997.

McCarthy, J. B., Skubitz, A. P., Iida, J., Mooradian, D. L., Wilke, M. S., \& Furcht, L. T. (1991). Tumor cell adhesive mechanisms and their relationship to metastasis. Seminars in Cancer Biology, 2(3), 155-167. PMid:1912525.

Natali, P., Nicotra, M. R., Cavaliere, R., Bigotti, A., Romano, G., Temponi, M., \& Ferrone, S. (1990). Differential expression of intercellular adhesion molecule 1 in primary and metastatic melanoma lesions. Cancer Research, 50(4), 1271-1278. PMid:1967552.

Park, S. H., Park, J. H. Y., Kang, J. S., \& Kang, Y. H. (2003). Involvement of transcription factors in plasma HDL protection against TNF$\alpha$-induced vascular cell adhesion molecule- 1 expression. The International Journal of Biochemistry \& Cell Biology, 35(2), 168-182. http://dx.doi.org/10.1016/S1357-2725(02)00173-5. PMid:12479867.

Rajavashisth, T. B., Andalibi, A., Territo, M. C., Berliner, J. A., Navab, M., Fogelman, A. M., \& Lusis, A. J. (1990). Induction of endothelial cell expression of granulocyte and macrophage colony-stimulating factors by modified low-density lipoproteins. Nature, 344(6263), 254-257. http://dx.doi.org/10.1038/344254a0. PMid:1690354.

Rice, G. E., \& Bevilacqua, M. P. (1989). An inducible endothelial cell surface glycoprotein mediates melanoma adhesion. Science, 246(4935), 1303-1306. http://dx.doi.org/10.1126/science.2588007. PMid:2588007.
Sen, C. K., \& Bagchi, D. (2001). Regulation of inducible adhesion molecule expression in human endothelial cells by grape seed proanthocyanidin extract. Molecular and Cellular Biochemistry, 216(1-2), 1-7. http:// dx.doi.org/10.1023/A:1011053300727. PMid:11216853.

Shikov, A. N., Pozharitskaya, O. N., \& Makarov, V. G. (2016). Aralia elata var. mandshurica (Rupr. \& Maxim.) J.Wen: An overview of pharmacological studies. Phytomedicine, 23(12), 1409-1421. http:// dx.doi.org/10.1016/j.phymed.2016.07.011. PMid:27765361.

Tak, P. P., Thurkow, E. W., Daha, M. R., Kluin, P. M., Smeets, T. J., Meinders, A. E., \& Breedveld, F. C. (1995). Expression of adhesion molecules in early rheumatoid synovial tissue. Clinical Immunology and Immunopathology, 77(3), 236-242. http://dx.doi.org/10.1006/ clin.1995.1149. PMid:7586733.

Tomatsu, M., Ohnishi-Kameyama, M., \& Shibamoto, N. (2003). Aralin, a new cytotoxic protein from Aralia elata, inducing apoptosis in human cancer cells. Cancer Letters, 199(1), 19-25. http://dx.doi. org/10.1016/S0304-3835(03)00348-3. PMid:12963119.

Tsujisaki, M., Imai, K., Hirata, H., Hanzawa, Y., Masuya, J., Nakano, T., Sugiyama, T., Matsui, M., Hinoda, Y., \& Yachi, A. (1991). Detection of circulating intercellular adhesion molecule- 1 antigen in malignant diseases. Clinical and Experimental Immunology, 85(1), 3-8. http:// dx.doi.org/10.1111/j.1365-2249.1991.tb05673.x. PMid:1712683.

Wilcox, J. N., Smith, K. M., Schwartz, S. M., \& Gordon, D. (1989). Localization of tissue factor in the normal vessel wall and in the atherosclerotic plaque. Proceedings of the National Academy of Sciences of the United States of America, 86(8), 2839-2843. http:// dx.doi.org/10.1073/pnas.86.8.2839. PMid:2704749.

Yu, L., Jinfeng, L., Zhenhong, L., Yanju, L., Mingna, L., \& Jing, J. (2011). The antitumor effects of araloside A extracted from the root bark of Aralia elata on human kidney cancer cell lines. African Journal of Pharmacy and Pharmacology, 5(4), 462-467. http://dx.doi. org/10.5897/AJPP10.317.

Zhang, Y., Peng, Y., Li, L., Zhao, L., Hu, Y., Hu, C., \& Song, S. (2013). Studies on cytotoxic triterpene saponins from the leaves of Aralia elata. Food Chemistry, 138(1), 208-213. http://dx.doi.org/10.1016/j. foodchem.2012.10.041. PMid:23265478. 